\title{
Sequencing of complete mitochondrial genome of brown algal Saccharina sp. ye- $\mathrm{C} 12$
}

\author{
Dong $\mathrm{Xu}^{\mathrm{a}, \mathrm{b}}$, Xiao Fan ${ }^{\mathrm{b}}$, Shuai Wang ${ }^{\mathrm{b}}$, Le $\mathrm{X \textrm {u } ^ { \mathrm { b } }}$, Xiaowen Zhang ${ }^{\mathrm{b}}$, Naihao $\mathrm{Ye}^{\mathrm{b}, \mathrm{c}}$ and Zhimeng Zhuang ${ }^{\mathrm{b}}$
}

${ }^{a}$ Fisheries College, Ocean University of China, Qingdao, China; ${ }^{b}$ Yellow Sea Fisheries Research Institute, Chinese Academy of Fishery Sciences, Qingdao, China; 'Function Laboratory for Marine Fisheries Science and Food Production Processes, Qingdao National Laboratory for for Marine Science and Technology, China

\section{ABSTRACT}

The complete sequence $(37654 \mathrm{bp}$ ) of the mitochondrial DNA (mtDNA) of the Saccharina sp. ye-C12 was determined using Illumina sequencing data. The genome contains 38 protein-coding genes (PCG), three ribosomal RNA (rRNA), 25 transfer RNA (tRNA) genes and the phylogenetic analysis based on the mitochondrial genomes of brown algae indicated that Saccharina sp. ye-C12 and Saccharina japonica are the most closely related species, which strongly supports their close phylogenetic affinity.

\section{ARTICLE HISTORY}

Received 8 December 2015 Revised 22 December 2015 Accepted 30 December 2015

\section{KEYWORDS}

Complete mitochondrial genome; illumina sequencing; saccharina $\mathrm{sp}$. ye-C12
Recently, the genome of S. japonica has been sequenced (Ye et al. 2015), which would powerfully push the genetic improvement of the species. In this study, strains of Saccharina collected from different area all over the world were resequenced, which make us have opportunity to uncover some mitochondrial genomes of these strains to develop more genetic tools due to the enough illumina sequencing data. In this study, the complete mitochondrial

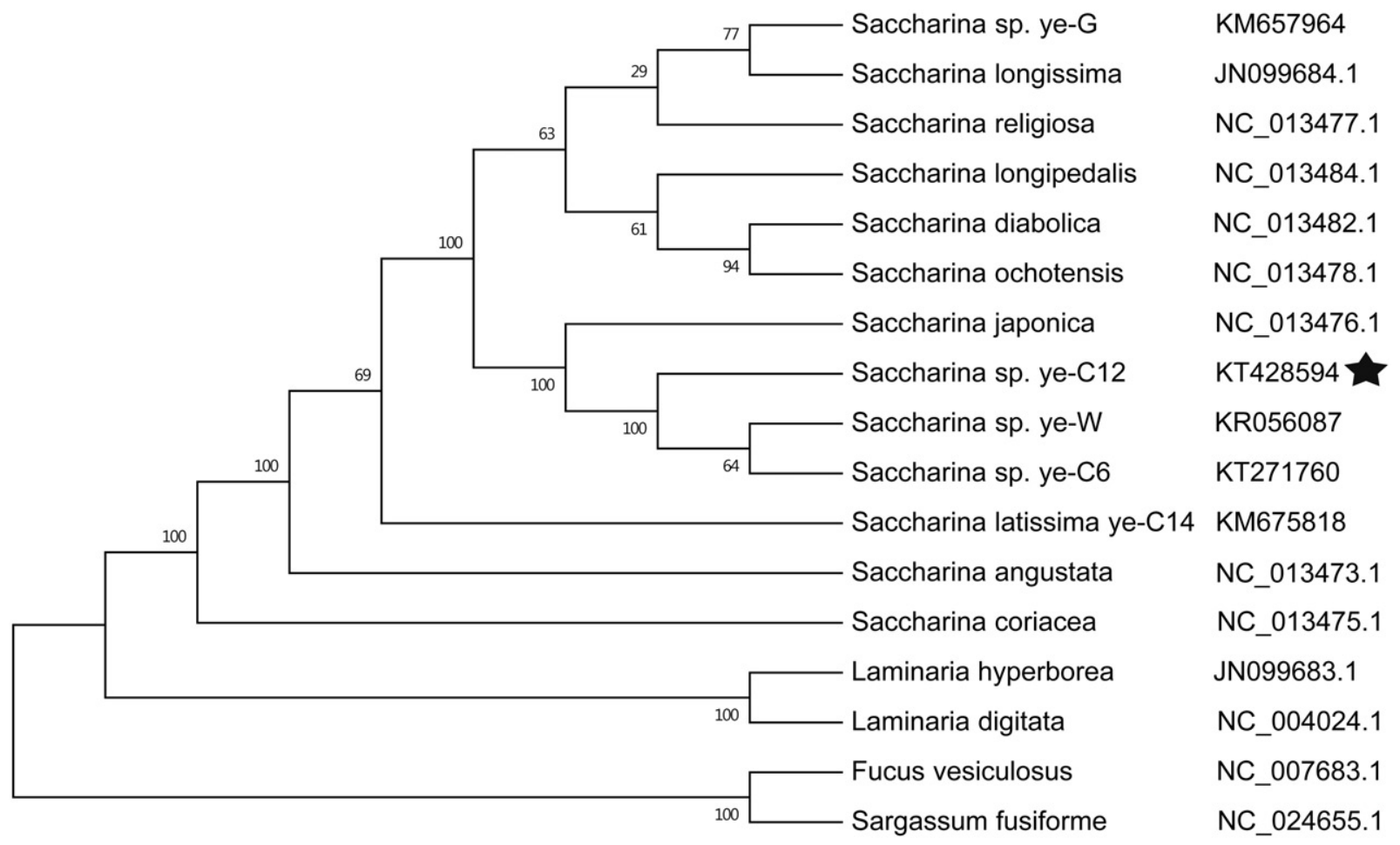

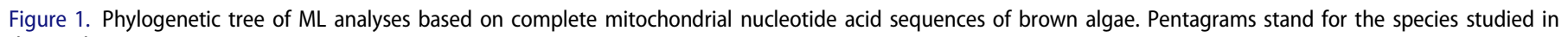
this work 
genome of a wild strain, sampled in Qingdao, China $\left(36^{\circ} 10^{\prime} \mathrm{N}\right.$, $\left.120^{\circ} 16^{\prime} 60\right)$ and stored in Yellow Sea Fisheries Research Institute Algae Culture Centre (YSFRIACC), Qingdao, China, is uncovered and named as Saccharina sp. ye-C12 (YSFRIACC accession number NO. YSFRI-Saccharina-sp-ye-C12, NCBI accession number NO. KT428594) based on the phylogenetic analysis with other complete brown algae mitochondrial genomes. Assembly and annotation were performed by the reference of our previous study (Guan et al. 2014).

The length of complete of Saccharina sp. ye-C12 is 37,654 bp and the genome contains 38 protein-coding genes (rps2-4, rps7-8, rps10-14, rps19, atp6, atp8, atp9, cox1-3, nad1-7, nad9, nad11, nad4L, rpl2, rpl5, rpl6, rpl14, rpl16, rp/31, ORF41, ORF130, ORF377, tatC and cob), 25 transfer RNA (tRNA) genes, three ribosomal RNA (rRNA) genes (5S rRNA, 16S rRNA and 23S rRNA). All 38 protein-coding genes (PCGs) have typical initiation codons (ATG). The numbers of PCGs that have complete termination codons TAA, TAG, TGA are 26, 8 and 4, respectively. No incomplete stop codons were found. Nucleotide frequency of the $\mathrm{H}$-strand is as follows: $\mathrm{T}, 36.29 \% ; \mathrm{A}, 28.41 \%$; , 14.72\%; and G, 20.58\%. Excluding the stop codons, the mitogenome of Saccharina sp. ye-C12 encodes 9637 amino acids. All the 25 typical tRNAs, ranging from 71 to 88 . The rRNAs of the 5S rRNA, 16S rRNA and 23S rRNA genes are $133 \mathrm{bp}, 1535$ bp and $2742 \mathrm{bp}$ in length, respectively.

Phylogenetic analysis shows that Saccharina sp. ye-C12 belongs to a Saccharina clade and is closely with $S$. japonica. This result is consistent with recent phylogenetic analyses and certain morphological characters (Zhang et al. 2013; Guan et al. 2014), indicating the related relationship and the proposal common source of the cultured kelp in China. Complete mitochondrial genomes enhanced the resolution and statistical confidence of the phylogenetic analysis (Figure 1).

\section{Disclosure statement}

The authors report no conflicts of interest.

\section{Funding information}

This work was supported by Scientific Research Funds for Central Nonprofit Institutes, Yellow Sea Fisheries Research Institutes (20603022015004), National Basic Research Special Foundation of China (2013FY110700), the Science Fund for Distinguished Young Scholars of Shandong Province (JQ201509) the program of leading talents of Qingdao (13-CX-27).

\section{References}

Guan Z, Fan X, Wang S, Dong X, Xiaowen Z, Dongsheng W, Yu M, Naihao Y. 2014. Sequencing of complete mitochondrial genome of brown algal Saccharina sp. ye-G. Mitochondrial DNA. 2014:1-2.

Ye N, Zhang X, Miao M, Fan X, Zheng Y, Xu D, Wang J, Zhou L, Wang D, Gao Y, et al. 2015. Saccharina genomes provide novel insight into kelp biology. Nature communications. 6. DOI: 10.1038/ncomms7986

Zhang J, Wang X, Liu C, Jin Y, Liu T. 2013. The complete mitochondrial genomes of two brown algae (Laminariales, Phaeophyceae) and phylogenetic analysis within Laminaria. J Appl Phycol. 25:1247-1253. 